\title{
ON PAIRWISE SUPER CONTINUOUS
} MAPPINGS IN BITOPOLOGICAL SPACES

\author{
F.H. KHEDR and A.M. ALSHIBANI \\ Department of Mathematics \\ College of Sciences \\ Girls Colleges General Adm. \\ P.O. Box 838, Dammam \\ Kingdom of Saudi Arabia \\ (Received June 25, 1990 and in revised form January 1, 1991)
}

\begin{abstract}
The aim of this paper is to define and study super-continuous mappings and some other forms of continuity such as strong continuity, perfect continuity and complete continuity in bitopological spaces and investigate the relations between these kinds of continuity and their effects on some kinds of spaces.
\end{abstract}

KEY WORDS AND PHRASES: $i j$-Super-continuous mapping, $i j$-almost open mapping, $i j$-almost closed mapping, $i j$-almost continuous mapping, $i j-\delta$-continuous mapping. 1980 AMS SUBJECT CLASSIFICATION CODE. 54E55.

\section{INTRODUCTION.}

The study of bitopological spaces was first initiated by J.C. Kelly [1] and thereafter a large number of papers have been done to generalize the topological concepts to bitopological setting. Munshi and Bassan [2] introduced a strong form of continuity, called super-continuous mappings and studied some of their properties in topological spaces. Noiri [3] obtained further.properties of super-continuous mappings and investigated the relation between super-continuity and several strong forms of continuity such as $\delta$-continuity and strongly $\theta$-continuity which was introduced by Noiri [4], and completely continuity which was given by Arya and Gupta [5]. Bose and Sinha [6] defined almost continuity in bitopological spaces. Banerjee [7] defined $\delta$-continuous and strong $\theta$-continuous mapping in these spaces. They study these mappings and some of their results on different kinds of spaces such as nearly compact, regular, almost regular and semi-regular spaces. The purpose of this paper is to define super-continuous mappings in bitopological spaces and investigate some of their properties and relations with other forms of continuity and its effects on some kinds of spaces.

Throughout this paper, by a space $X$ we mean a bitopological space $\left(X, \tau_{1}, \tau_{2}\right)$. By $i-$ int $A$ and $i-\operatorname{cl} A$ we shall mean the interior and the closure of a subset $A$ of $X$ with respect to $\tau_{1}$, respectively, where $i, j=1$ or 2 and $i \neq j$.

A subset $S$ of $X$ is said to be $i j$-regular open (resp. ij-regular closed) if $i-i n t(j-c l S)=S$ (resp. $i-c l(j-i n t S)=S)$. $S$ is said to be pariwise regular open (resp. pairwise regular closed) if it is both $i j$-regular open and $j i$-regular open (resp. $i j$-regular closed and $j i$-regular closed), denoted by

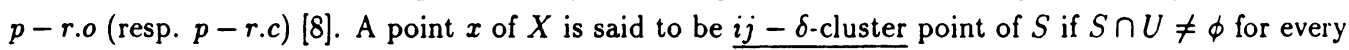


$i j-r . o$ set $U$.containing $x$. The set of all $i j-\delta$ cluster points of $S$ is called $2 j-\delta$-closure of $S$ and is denoted by $i j-c l_{\delta}(S)$. A subset $S$ of $X$ is said to be $i j-\delta$-closed if $i j-\delta$-cluster points of $S \subset S$. The complement of $i j-\delta$-closed set is $i j-\delta$-open. So a set is $i j-\delta$-open if it is explessible as a union of $i j-r . o$ sets. $S$ is said to be pairwise- $\delta$-closed (resp. pairwise- $\delta$-open) if it is both ij- $\delta$-closed and $j i-\delta$-closed (resp. $i j-\delta$-open, and $j i-\delta$-open) and will be denoted by $p-\delta$-closed (resp. $p-\delta$-open).

A bitopological space $X$ is said to be $i j$-semi regular [8] (resp. ij-regular [1], ij- almost regular [9]) iff for each $x \in X$ and for each $i$-open set $V$ of $X$, there is an $i$-open set $U$ containing $x$ such that $x \in U \subset i-\operatorname{int}(j-c l U) \subset V$ (resp. $x \in U \subset j-c l U \subset V, x \in U \subset j-c l U \subset i-\operatorname{int}(j-c l V))$. $X^{\prime}$ is pairwise-semi regular (resp. pairwise-regular, pariwise almost regular) if it is both ij-semi regular and $j i$-semi regular) (resp. $i j$-regular and $j i$-regular, $i j$-almost regular and $j i$-almost regular).

A subset $S$ of a bitopological space $\left(X, \tau_{1}, \tau_{2}\right)$ is said to be ij-nearly compact relative to $X[7]$ iff each $i$-open cover $\mathcal{U}$ of $S$ has a finite subcollection $\mathcal{U}_{0}$ such that $S \subset \cup_{U \in U_{0}} i-i n t(j-c l U)$.

\section{SUPER-CONTINUOUS MAPPINGS}

DEFINITION 2.1. A mapping $f:\left(X, \tau_{1}, \tau_{2}\right) \longrightarrow\left(Y, \sigma_{1}, \sigma_{2}\right)$ is said to be $\underline{i j \text {-super continuous }}$ at a point $x \in X$ if every $i$-neighborhood $V$ of $f(x)$ there exists an $i$-neighborhood $U$ of $X$ such that $\overline{f(i-i n t}(j-c l U)) \subset V$. A mapping $f$ is said to be $i j$-super continuous (denoted by $i j-S C$ ) if it is $i j$-super continuous at each point of $X$ and it is said to be pairwise $S C$ if it is both $i j-S C$ and $j i-S C$.

REMARK 2.1. It is clear that if $f$ is $i j$-super continuous at $x \in X$ then it is $i$-continuous at $x$. But the converse is not true as seen from the following example.

EXAMPLE 2.1. Let $X=Y=\{a, b, c\}$ and $\tau_{1}=\{\phi, X,\{a\},\{b, c\},\{a, b\},\{b\}\}, \tau_{2}=\{\phi, X,\{b\},\{b, c\}\}$, $\sigma_{1}=\{\phi, Y,\{a\},\{b, c\}\}, \sigma_{2}=\{\phi, Y,\{b\},\{b, c\}\}$, and let $f:\left(X, \tau_{1}, \tau_{2}\right) \longrightarrow\left(Y, \sigma_{1}, \sigma_{2}\right\}$ be given by $f(x)=x$ for each $x \in X$, then $f$ is 1 -continuous and 2-continuous but it is not 12 -super continuous at $x=c$ since if $x=c$ then $f(x)=c$, let $V=\{b, c\}$, then there exists no 1-neighborhood $U$ of $c$ such that $f(1-\operatorname{int}(2-c l U)) \subset V$.

THEOREM 2.1. For a mapping $f:\left(X, \tau_{1}, \tau_{2}\right) \longrightarrow\left(Y, \sigma_{1}, \sigma_{2}\right)$ the following are equivalent:

(a) $f$ is $i j-S C$.

(b) Inverse image of every $i$-open subset of $Y$ is an $i j-\delta$-open subset of $X$.

(c) Inverse image of every $i$-closed subset of $Y$ is an $i j$ - $\delta$-closed subset, of $X$.

(d) For each point $x$ of $X$ and each $i$-neighborhood $V$ of $f(x)$ there exists an $i j-\delta$-open neighborhood $U$ of $x$ such that $f(U) \subset V$.

PROOF. (a) $\longrightarrow$ (b). Let $A$ be any $i$-open subset of $Y$ and $x \in f^{-1}(A)$. Then $f(x) \in A$ and so there exists an $i$-neighborhood $U$ of $x$ such that $x \in U$ and $f(i-\operatorname{int}(j-c l U)) \subset A$. So, $x \in i-\operatorname{int}(j-c l U)=V \subset f^{-1}(A)$. But $V$ is $i j-r . o$, so $f^{-1}(A)$ is expressible as an arbitrary union of $i j$-regular open sets, hence $f^{-1}(A)$ is $i j-\delta$-open.

(b) $\longrightarrow$ (c). Obvious.

$\left(\right.$ c) $\longrightarrow$ (d). Let $V$ be an $i$-neighborhood of $f(x)$ so $Y \sim V$ is $i$-closed by (c), $f^{-1}(Y \sim V)$ is $i j-\delta$-closed, hence $f^{-1}(V)$ is $i j-\delta$-open; so we have $x \in f^{-1}(V)=U$ and $f(U) \subset V$, where $U$ is $i j-\delta$-open.

(d) $\longrightarrow$ (a). For each $x \in X$ and each $i$-open neighborhood $V$ of $f(x)$ there exists an $i j-\delta$-open neighborhood $U$ of $x$ such that $f(U) \subset V$. But $U$ is $i j-\delta$-open, so there exists an $i j-$ r.o set 0 such that $x \in 0 \subset U$, hence $f(x) \subset f(0)=f(i-\operatorname{int}(j-c l 0)) \subset f(U) \subset V$. So $f$ is ij-SC. 
THEOREM 2.2. Let $f:\left(X, \tau_{1}, \tau_{2}\right) \longrightarrow\left(Y, \sigma_{1}, \sigma_{2}\right)$ be an $i$-continuous mapping of an ij-semi regular space $X$ into $Y$, then $f$ is $i j-S C$.

PROOF. Let $G$ be an $i$-neighborhood of $f(x)$, so $f^{-1}(G)$ is an $i$-neighborhood of $x$. Since $X$ is $i j$-semi regular, there exists an $i$-open set $V$ such that $x \in V \subset i-\operatorname{int}(j-c l V) \subset f^{-1}(G)$. So $f(i-\operatorname{int}(j-c l V)) \subset G$ and hence $f$ is $i j-S C$.

THEOREM 2.3. Let $X$ and $Y$ be bitopological spaces, then a mapping $f:\left(X, \tau_{1}, \tau_{2}\right) \longrightarrow\left(Y, \sigma_{1}, \sigma_{2}\right)$ is $i j-S C$ iff the inverse image under $f$ of every member of $i$-base for $Y$ is $i j-\delta$-open.

PROOF. From Theorem 2.1 parts (a) and (b).

THEOREM 2.4. A mapping $f:\left(X, \tau_{1}, \tau_{2}\right) \longrightarrow\left(Y, \sigma_{1}, \sigma_{2}\right)$ is $i j-S C$ iff $f\left(i j-c l_{\delta}(A)\right) \subset \imath-c l(f(A))$, for each subset $A$ of $X$.

PROOF. Let $f$ be $i j-S C$, since $i-c l f(A)$ is an $i$-closed subset of $Y$, then $f^{-1}(i-c l f(A))$ is $i j-\delta$-closed in $X$. But since $f(A) \subset i-c l f(A)$, then $A \subset f^{-1}(i-c l f(A))$ and so $i j-c l_{\delta} A \subset$ $i j-c l_{\delta}\left(f^{-1}(i-c l f(A))\right)=f^{-1}(i-c l f(A))$. Hence $f\left(i j-c l_{\delta} A\right) \subset i-c l f(A)$.

Conversely, let $f\left(i j-c l_{\delta} A\right) \subset i-c l f(A)$ for each $A \subset X$ and let $F$ be any $i$-closed subset of $Y$, so $i-c l F=F$. Since $f^{-1}(F) \subset X$, so $f\left(i j-c l_{\delta} f^{-1}(F)\right) \subset i-c l f f^{-1}(F) \subset i-c l F=F$ and $i j-c l_{\delta} f^{-1}(F) \subset f^{-1}(F)$. Then $f^{-1}(F)$ is $i j-\delta$-closed and by Theorem $2.1 f$ is $i j-S C$.

THEOREM 2.5. A mapping $f:\left(X, \tau_{1}, \tau_{2}\right) \longrightarrow\left(Y, \sigma_{1}, \sigma_{2}\right)$ is $i j-S C$ iff $i \jmath-c l_{\delta} f^{-1}(B) \subset f^{-1}(2-c l B)$ for each $B \subset Y$.

PROOF: Let $f$ be $i j-S C$. Since $i-c l B$ is $i$-closed subset of $Y$, then $f^{-1}(i-c l B)$ is $i j-\delta$ closed in $X$ and since $B \subset i-c l B$; then $i j-c l_{\delta} f^{-1}(B) \subset i j-c l_{\delta} f^{-1}(i-c l B)=f^{-1}(i-c l B)$ and so $i j-c l_{\delta} f^{-1}(B) \subset f^{-1}(i-c l B)$.

Conversely, let $i j-c l_{\delta} f^{-1}(B) \subset f^{-1}(i-c l B)$ for each $B \subset Y$ and let $F$ be an i-closed subset of $Y$. Then $i j-c l_{\delta} f^{-1}(F) \subset f^{-1}(i-c l F)=f^{-1}(F)$, but since $f^{-1}(F) \subset i j-c l_{\delta} f^{-1}(F)$, so $f^{-1}(F)=i j-c l_{\delta} f^{-1}(F)$ and hence $f^{-1}(F)$ is $i j-\delta$-closed. Therefore $f$ is $i j-S C$.

DEFINITION 2.2. A mapping $f:\left(X, \tau_{1}, \tau_{2}\right) \longrightarrow\left(Y, \sigma_{1}, \sigma_{2}\right)$ is said to be $i j$-almost open if the image of every $i j$-regular open subset of $X$ is $i$-open in $Y . f$ is said to be $i j$-almost closed if the image of every $i j$-regular closed subset of $X$ is $i$-closed in $Y$.

DEFINITION 2.3. [6] A mapping $f:\left(X, \tau_{1}, \tau_{2}\right) \longrightarrow\left(Y, \sigma_{1}, \sigma_{2}\right)$ is said to be $i j$-almost continuous at a point $x \in X$ if every $i$-neighborhood $V$ of $f(x)$ there exists an $i$-neighborhood $U$ of $x$ such that $f(U) \subset i-\operatorname{int}(j-c l V)$.

THEOREM 2.6. If $f:\left(X, \tau_{1}, \tau_{2}\right) \longrightarrow\left(Y, \sigma_{1}, \sigma_{2}\right)$ is $i j$-almost open and $i j-S C$ mapping of $X$ onto $Y$ and if $g:\left(Y, \sigma_{1}, \sigma_{2}\right) \longrightarrow\left(Z, \gamma_{1}, \gamma_{2}\right)$ is a mapping of $Y$ into $Z$, then $g$ of is ij $-S C$ iff $g$ is $i$-continuous.

PROOF. Let $\mathrm{f}$ be an $i j$-almost open $i j-S C$ mapping, and let $g$ be $i$-continuous. Let $U$ be an $i$-closed subset of $Z$, consider $f^{-1}\left(g^{-1}(U)\right)$, since $U$ is $i$-closed in $Z$ and $g$ is $i$-continuous, so $g^{-1}(U)$ is $i$-closed in $Y$. Also, since $f$ is $i j-S C$, so $f^{-1}\left(g^{-1}(U)\right)$ is $i j-\delta$-closed subset of $X$, hence $f \circ g$ is $i j-S C$.

Conversely, let $g$ of be $i j-S C$, then for each $i$-open subset $G$ of $Z(g \circ f)^{-1}(G)$ is $i j-\delta$-open subset of $X$. Since $f$ is $i j$-almost open and onto, then $f\left[f^{-1}\left(g^{-1}(G)\right]=(g \circ f)^{-1}(G)\right]=g^{-1}(G)$ is $i$-open subset of $Y$, hence $g$ is $i$-continuous.

THEOREM 2.7. Let $X, Y$, and $Z$ be bitopological spaces and let $f:\left(X, \tau_{1}, \tau_{2}\right) \longrightarrow\left(Y, \sigma_{1}, \sigma_{2}\right)$ be $i j$-almost continuous and $g:\left(Y, \sigma_{1}, \sigma_{2}\right) \longrightarrow\left(Z, \gamma_{1}, \gamma_{2}\right)$ be $i j-S C$, then $g$ of $:\left(X, \tau_{1}, \tau_{2}\right) \longrightarrow\left(Z, \gamma_{1}, \gamma_{2}\right)$ is $i$-continuous.

PROOF: Let $x \in X$, so $f(x) \in Y$, consider $(g \circ f)(x)$. Let $U$ be an $i$-neighborbood of $(g \circ f)(x)$, since $g$ is $i j-S C$ so $g^{-1}(U)$ is $i j-\delta$-open subset of $Y$, so there exists an $i j-r$.o subset $V$ of $Y$ such that $f(x) \in V \subseteq g^{-1}(U)$, but since $f$ is $i j$-almost continuous, then there exists an $i$-neighborhood $N$ of $x$ such that $f(N) \subset V \subset g^{-1}(U)$. Then $f^{-1}(f(N)) \subset f^{-1}\left(g^{-1}(U)\right)$ and $N \subset f^{-1}\left(g^{-1}(U)\right)$; so 
$(g \circ f)^{-1}(U)$ is an $i$-open subset $f X$, hence $g \circ f$ is $i$-continuous.

REMARK 2.2. If $f:\left(X, \tau_{1}, \tau_{2}\right) \longrightarrow\left(Y, \sigma_{1}, \sigma_{2}\right)$ is $i j$-almost continuous and $g$ of $:\left(X, \tau_{1}, \tau_{2}\right) \longrightarrow$ $\left(Z, \gamma_{1}, \gamma_{2}\right)$ is $i$-continuous, then $g:\left(Y, \sigma_{1}, \sigma_{2}\right) \longrightarrow\left(Z, \gamma_{1}, \gamma_{2}\right)$ need not be $i j-S C$ as seen from the following exmaple.

EXAMPLE 2.2. Let $X=R$ with $\tau_{1}=\{\phi, R$, complement of countable subsets of $R\} . \tau_{2}=\{\phi, R\}$, and $Y=\{a, b\}$ with $\sigma_{1}=\{Y, \phi,\{a\}\}, \sigma_{2}=\{Y, \phi\}$, and $Z=\{1,2\}$ with $\gamma_{1}=\{Z, \phi,\{2\}\}, \gamma_{2}=$ $\{Z, \phi,\{1\}\}$, let $f:\left(X, \tau_{1}, \tau_{2}\right) \longrightarrow\left(Y, \sigma_{1}, \sigma_{2}\right)$ defined by

$$
\begin{aligned}
f(x) & =a & & \text { if } x \text { is irrational } \\
& =b & & \text { if } x \text { is rational }
\end{aligned}
$$

and $g:\left(Y, \sigma_{1}, \sigma_{2}\right) \longrightarrow\left(Z, \gamma_{1}, \gamma_{2}\right)$ defined by $g(a)=2, g(b)=1$, then $f$ is 12 -almost continuous and $g \circ f$ is 1 -continuous but $g$ is not $12-S C$, since if $x=a$, then $g(a)=2$, let $V=\{2\}$ then there is no 1 -neighborhood $U$ of $\{a\}$ such that $g(1-\operatorname{int}(2-c l U)) \subset V$.

DEFINITION 2.4. [7] A mapping $f:\left(X, \tau_{1}, \tau_{2}\right) \rightarrow\left(Y, \sigma_{1}, \sigma_{2}\right)$ is said to be ij- $\delta$-continuous if for each $x$ in $X$ and each $i$-neighborhood $V$ of $f(x)$ there exists an $i$-neighborhood $U$ of $x$ such that $f(i-\operatorname{int}(j-c l U)) \subset i-\operatorname{int}(j-c l V)$.

REMARK 2.3. For a mapping $f:\left(X, \tau_{1}, \tau_{2}\right) \longrightarrow\left(Y, \sigma_{1}, \sigma_{2}\right)$, we have the following implications:

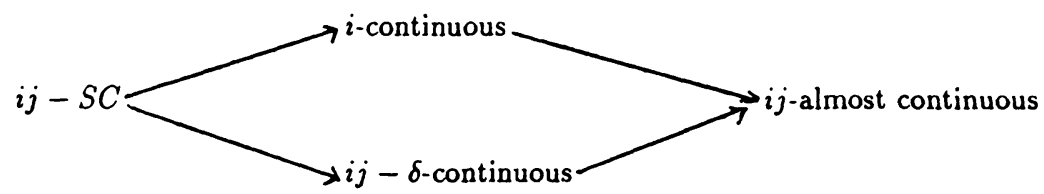

The converse may not be true as seen from Example 2.1 in [6] and Example 2.1.

THEOREM 2.8. Let $X$ and $Y$ be $i j$-semi regular spaces, then for a mapping $f:\left(X, \tau_{1}, \tau_{2}\right) \longrightarrow$ $\left(Y, \sigma_{1}, \sigma_{2}\right)$ the following properties are equivalent:

(a) $i j-S C$

(b) i-continuous

(c) $i j-\delta$-continuous

(d) $i j$-almost continuous

PROOF. We shall only prove that $(d) \longrightarrow(a)$. Let $f$ be $i j$-almost continuous and let $x \in X$ and $y=f(x)$, since $Y i j$-semi regular, for every $i$-open neighborhood $V$ of $y$ there exists an $i$-open neighborhood $V^{\prime}$ of $y$ such that $f(x)=y \in V^{\prime} \subset\left(i-i n t\left(j-c l V^{\prime}\right)\right) \subset V$. Since $f$ is $i j$-almost continuous, there exists an $i$-open neighborhood $U$ of $x$ such that $f(U) \subset i-i n t\left(j-c l V^{\prime}\right) \subset V$. By $i j$. semi regularity of $X$ there exists an $i$-open neighborhood $U^{\prime}$ of $x$ such that $x \in U^{\prime} \subset i-i n t\left(j-c l U^{\prime}\right) \subset$ $U$. Hence we have $f\left(i-i n t\left(j-c l U^{\prime}\right)\right) \subset V$ and so $f$ is $i j-S C$.

THEOREM 2.9. Let $f:\left(A, P_{1}, P_{2}\right) \longrightarrow\left(X \times Y, Q_{1}, Q_{2}\right)$ be given by the equation $f(a)=$ $\left(f_{1}(a), f_{2}(a)\right)$ for every $a$ in $A$, then $f$ is $i j-S C$ iff $f_{1}:\left(A, P_{1}, P_{2}\right) \longrightarrow\left(X, \tau_{1}, \tau_{2}\right)$ and $f_{2}:\left(A, P_{1}, P_{2}\right) \longrightarrow$ $\left(Y, \sigma_{1}, \sigma_{2}\right)$ are $i j-S C$.

PROOF. Let $f_{1}, f_{2}$ be $i j-S C$, let $a \in A$ and $Q=U_{1} \times U_{2}$ be an $i$-open subset of $X \times Y$ such that $f(a)=\left(f_{1}(a), f_{2}(a)\right) \in U_{1} \times U_{2}$, so $f_{1}(a) \in U_{1}, f_{2}(a) \in U_{2}$, where $U_{1}$ is an $i$-open subset of $X$ and $U_{2}$ is an $i$-open subset of $Y$, but since $f_{1}, f_{2}$ are $i j-S C$ so there exists $i j-r . o$ sets $U_{1}^{\prime}$ and $U_{2}^{\prime}$ in $A$ such that $f_{1}\left(U_{1}^{\prime}\right) \subset U_{1}, f_{2}\left(U_{2}^{\prime}\right) \subset U_{2}$. Put $U^{\prime}=U_{1}^{\prime} \cap U_{2}^{\prime}, U^{\prime}$ is $i j-r . o$ and $f\left(U^{\prime}\right) \subset\left(f_{1}\left(U_{1}^{\prime}\right), f_{2}\left(U_{2}^{\prime}\right)\right) \subset U_{1} \times U_{2}$. Hence $f$ is $i j-S C$. 
Conversely, let $f$ be $i j-S C$ and let $a \in A$ and $U_{l}$ be an i-open subset of $X$ containing $f_{1}(a)$, then $U_{l} \times Y$ is an $i$-open subset of $X \times Y$ containing $f(a)$.

Since $f$ is $i j-S C$, there exists an $i j-r . o$ subset $V$ of $A$ containing a such that $f(V) \subset U_{l} \times Y$ and so $\left(f_{1}(V), f_{2}(V)\right) \subset U_{1} \times Y$.

Then $f_{1} \subset U_{1}$ and therefore $f_{1}$ is $i j-S C$. In a similar way we can prove that $f_{2}$ is ij $-S C$.

THEOREM 2.10. Let $f:\left(X, \tau_{1}, \tau_{2}\right) \longrightarrow\left(Y, \sigma_{1}, \sigma_{2}\right)$ be a mapping and $g:\left(X, \tau_{1}, \tau_{2}\right) \longrightarrow(X \times$ $\left.Y, P_{1}, P_{2}\right)$ given by $g(x)=(x, f(x))$ for all $x$ in $X$, be the graph mapping, then $g$ is $i j-S C$ iff $f$ is $i j-S C$ and $X$ is $i j$-semi regular.

PROOF. Let $g$ be $i j-S C$ and $U$ be an $i$-open subset of $X$ containing $x$, so $U \times Y$ is an $i$-open subset of $X \times Y$ containing $g(x)$. Since $g$ is $i j-S C$, there exists an $i j-r . o$ subset $W$ of $X$ containing $x$ such that $g(W) \subset U \times Y$. Then $x \in W \subset g^{-1}(U \times Y) \subset U$ and therefore $X$ is $i j$-semi regular from Theorem 2.9 since $g$ is $i j-S C$ and $g(x)=(x, f(x))$, so $f$ is $i j-S C$.

Conversely, let $f$ be $i j-S C$ and $X$ be $i j$-semi regular. For each $x$ in $X$, and each $i$-neighborhood $W$ of $g(x)$, there exists an $i$-neighborhood $U$ of $x$ and an $i$-neighborhood $V$ of $f(x)$ such that $(x, f(x)) \in$ $U \times V \subset W$.

Since $X$ is $i j$-semi regular, there exists an $i j-r . o$ subset $G_{1}$ of $X$ such that $x \in G_{1} \subset U$. Since $f$ is $i j-S C$, there exists an $i j-r . o$ subset $G_{2}$ of $X$ such that $x \in G_{2}$ and $f\left(G_{2}\right) \subset V$.

Let $G=G_{1} \cap G_{2}$. Then $G$ is an $i j-r . o$ subset of $X$ and $g(G) \subset U \times V \subset W$. Hence $g$ is $i j-S C$. 3. $i j$-NEARLY COMPACT SPACE AND $i j$-SUPER CONTINUOUS MAPPINGS.

THEOREM 3.1. A bitopological space $X$ is $i j$-nearly compact iff every $i j-\delta$-open cover of $X$ has a finite subcover.

PROOF. Let $X$ be $i j$-nearly compact and let $\mathcal{U}=\cup\left\{U_{\alpha} \mid \alpha \in \Delta\right\}$ be an $i j-\delta$-open cover of $X$. For each $U_{\alpha} \in \mathcal{U}$ and $x \in U_{\alpha}$ there exists an $i$-open set $V_{\alpha}$ such that $x \in V_{\alpha} \subset i-\operatorname{int}\left(j-c l V_{\alpha}\right) \subset U_{\alpha}$. Then $\left\{V_{\alpha} \mid \alpha \in \Delta\right\}$ is an $i$-open cover for $X$, so there is a finite subset $\Delta_{0}$ such that $X \subset \cup_{\alpha \in \Delta_{0}}{ }^{i-}$ $\operatorname{int}(j-c l V) \subset \cup_{\alpha \in \Delta_{0}} U_{\alpha}$. So $U$ has finite subcollection which covers $X$.

Coversely, let $U=U\left\{U_{\alpha} \mid \alpha \in \Delta\right\}$ be an $i$-open cover of $X$. Since $U_{\alpha} \subset \imath-i n t\left(j-c l U_{\alpha}\right)$, therefore $X \subset \cup_{\alpha \in \Delta} U_{\alpha} \subset U_{\alpha \in \Delta i}-\operatorname{int}\left(j-\operatorname{cl} U_{\alpha}\right)$. But $\cup_{\alpha \in \Delta^{i}} i-\operatorname{int}\left(j-c l U_{\alpha}\right)$ is an $i j-\delta$ open cover of $X$, so there exists a finite subset $\Delta_{0}$ of $\Delta$ such that $X \subset U_{\alpha \in \Delta_{0}} i-i n t\left(j-c l U_{\alpha}\right)$. Hence $X$ is ij-nearly compact.

COROLLARY 3.1. Any $i j$-regular closed subset of an $i j$-nearly compact space is $i j$-nearly compact.

PROOF. Obvious.

THEOREM 3.2, Let $f:\left(X, \tau_{1}, \tau_{2}\right) \longrightarrow\left(Y, \sigma_{1}, \sigma_{2}\right)$ be an $i j-S C$ mapping of an $i j$-nearly compact space $X$ into a bitopological space $Y$, then $f(X)$ is $i$-compact.

PROOF. Let $\left\{O_{\alpha}\right\}$ be any $i$-open cover of $f(X)$. Since $f$ is $i j-S C$, so $f^{-1}\left(O_{\alpha}\right)$ is $i j-\delta$. open in $X$ and $\cup f^{-1}\left(O_{\alpha}\right)$ is $i j-\delta$-open cover of $X$, hence $X=\cup_{i=1}^{n} f^{-1}\left(O_{\alpha_{1}}\right)=f^{-1}\left(\cup_{i=1}^{n} O_{\alpha_{1}}\right)$. So, $f(X)=f f^{-1}\left(\cup_{i=1}^{n} O_{\alpha}\right) \subset \cup_{i=1}^{n} O_{\alpha}$, hence $f(X)$ is $i$-compact.

DEFINITION 3.1. A subset $K$ of $X$ is called $i j-H$ set if for each cover $\left\{U_{\alpha}: \alpha \in \nabla\right\}$ of $K$ by $i$-open subsets of $X$ there exists a finite subset $\nabla_{0}$ of $\nabla$ such that $K \subset \cup\left\{j-c l U_{\alpha} \mid \alpha \in \nabla_{0}\right\}$.

DEFINITION 3.2. [1] A bitopological space $\left(X, \tau_{1}, \tau_{2}\right)$ is called $i j$-Hausdorff iff for each pair of distinct points $x, y$ of $X$, there are an $i$-open neighborhood $A$ of $x$ and a $j$-open neighborhood $B$ of $y$ such that $A \cap B=\phi$.

THEOREM 3.3. For a mapping $f:\left(X, \tau_{1}, \tau_{2}\right) \longrightarrow\left(Y, \sigma_{1}, \sigma_{2}\right)$ let:

(a) $f$ is $i j-S C$ and $Y$ is $i j$-Hausdorff.

(b) for every $(x, y) \notin G(f)$ there exists an $i j-r$.o subset $U$ of $X$ and $j i-r . o$ subset $V$ of $Y$ containing $x$ and $y$, respectively, such that $f(U) \cap i-c l V=\phi$ (where $G(f)$ is the graph of $f$ ).

(c) $f^{-1}(K)$ is $i j-\delta$-closed in $X$ for every $j i-H$ set $K$ of $Y$. Then (a) $\longrightarrow$ (b) $\longrightarrow$ (c). 
PROOF. (a) $\longrightarrow$ (b) Let $(x, y) \notin G(f)$, so $y \neq f(x)$ for $x \in X$. Since $Y$ is $i j$-Hausdorff, there exists an $i j-r . o$ subset $U$ and $j i-r . o$ subset $V$ of $Y$ such that $f(x) \in U, y \in V$ and $U \cap \imath-c l V=\phi$. But $f$ is $i j-S C$, so there exists an $i j-r$.o subset $U^{\prime}$ containing $x$ such that $f(U)^{\prime} \subset U$; hence $f\left(U^{\prime}\right) \cap i-c l V=\phi$.

(b) $\longrightarrow$ (c) Let $K$ be a $j i-H$ set of $Y$ and $x \notin f^{-1}(K)$, for every $y \in K, y \neq f(x)$ and $(x, y) \notin G(f)$, there exists an $i j-$ ro subset $U_{v}$ and an $j i-r . o$ subset $V_{y}$ such that $x \in U_{y}$ and $y \in V_{y}$ and $f\left(U_{y}\right) \cap i-c l V_{y}=\phi$. The family $\left\{V_{y} \mid y \in K\right\}$ is a $j$-open cover of $K$. Since $K$ is a $j i-H$ set, there exists a finite subset $K_{0}$ of $K$ such that $K \subset U\left\{i-c l V_{y} \mid y \in K_{0}\right\}$. Let $U=\cap\left\{U_{y} \mid y \in K_{0}\right\}$; so $U$ is $i j-r . o$ subset containing $x$ and $f(U) \cap K=\phi$. Then $U \cap f^{-1}(K)=\phi$, and $f^{-1}(K)$ is $i j-\delta$-closed in $X$.

\section{STRONG FORMS OF CONTINUITY.}

DEFINITION 4.1. A mapping $f:\left(X, \tau_{1}, \tau_{2}\right) \longrightarrow\left(Y, \sigma_{1}, \sigma_{2}\right)$ is said to be $j$-strongly continuous if $f(j-c l A) \subset f(A)$ for every subset $A$ of $X$.

COROLLARY 4.1. A function $f$ is $j$-strongly continuous iff $f^{-1}(B)$ is both $j$-open and $j$-closed in $X$ for every subset $B$ of $Y$.

PROOF. Let $B \subset Y$ and let $f^{-1}(B)=F \subset X$, since $f$ is $j$-strongly continuous, $f(j-c l F) \subset f(F)$; hence $j-c l F \subset F$; so $F$ is $j$-closed. Similarly $X \sim F \subset X$; hence $X \sim F$ is $j$-closed, so $F$ is $j$-open.

DEFINITION 4.2. A mapping $f:\left(X, \tau_{1}, \tau_{2}\right) \longrightarrow\left(Y, \sigma_{1}, \sigma_{2}\right)$ is said to be ij-clopen continuous (resp., $i j$-strongly $\theta$-continuous [7]) if for every $x \in X$ and for every $i$-open neighborhood $V$ of $f(x)$ there exists a $j$-closed and $i$-open neighborhood $U$ of $x$ (resp. i-open) such that $f(U) \subset V$ (resp. $f(j-c l U) \subset V)$.

DEFINITION 4.3. A mapping $f:\left(X, \tau_{1}, \tau_{2}\right) \longrightarrow\left(Y, \sigma_{1}, \sigma_{2}\right)$ is said to be $i j$-perfectly continuous (resp. ij-completely continuous) if $f^{-1}(V)$ is both $j$-closed and $j$-open (resp. ij-r.o) in $X$ for each $i$-open subset $V$ of $Y$.

DEFINITION 4.4. A mapping $f:\left(X, \tau_{1}, \tau_{2} \longrightarrow\left(Y, \sigma_{1}, \sigma_{2}\right)\right.$ is said to be an $i j-R$ map if $f^{-1}\left(V^{\prime}\right)$ is $i j-r . o$ for every $i j-r . o$ subset $V$ of $Y$.

REMARK 4.1 . It is obvious that $i j$-clopen continuous $\longrightarrow i j$-strongly $\theta$ - continuous $\longrightarrow i j-S C$ but the converse may not be true as can be seen from the follwoing example:

EXAMPLE 4.1. Let $X=Y=\{a, b, c, d\}$ and let $\tau_{1}=\{\phi, X,\{a\},\{b\},\{a, b\}\}, \tau_{2}=\{\phi, X,\{a\},\{b\}$, $\{a, b\},\{a, b, c\},\{a, c\}\}, \sigma_{1}=\{\phi, X,\{a\},\{a, c\},\{a, c, d\}\}, \sigma_{2}=\{\phi, X,\{b\},\{b, d\}\}$. Let $f:\left(X, \tau_{1}, \tau_{2}\right) \rightarrow$ $\left(Y, \sigma_{1}, \sigma_{2}\right)$ be defined by $f(a)=f(b)=a, f(c)=f(d)=b$. Then $f$ is $12-S C$ but it is not 12-strongly $\theta$-continuous.

THEOREM 4.1. If $f:\left(X, \tau_{1}, \tau_{2}\right) \longrightarrow\left(Y, \sigma_{1}, \sigma_{2}\right)$ is $i j-S C$ and $X$ is $i j$-almost regular, then $f$ is ij-strongly $\theta$-continuous.

PROOF. Let $x \in X$ and let $V$ be an $i$-open neighborhood of $f(x)$. Then there exists an $i j-r . o$ neighborhood $U$ of $X$ such that $f(U) \subset V$. Since $X$ is $i j$-almost regular, there exists an $i$-open neighborhood $U^{\prime}$ of $x$ such that $x \in U^{\prime} \subset j-c l U^{\prime} \subset U$. Then $f\left(j-c l U^{\prime}\right) \subset f(U) \subset V$ and so $f$ is $i j$-strongly $\theta$-continuous.

COROLLARY 4.2. If $X$ is $i j$-regular, then for a mapping $f:\left(X, \tau_{1}, \tau_{2}\right) \longrightarrow\left(Y, \sigma_{1}, \sigma_{2}\right)$ the following properties are equivalent:

(a) $f$ is $i j$-strongly $\theta$-continuous.

(b) $f$ is $i j-S C$.

(c) $f$ is $i$-continuous.

PROOF. Follows from Theorems 2.2 and 4.1 . 
COROLLARY 4.3. If $X$ and $Y$ are $i j$-regular, then for a mapping $f:\left(X, \tau_{1}, \tau_{2}\right) \longrightarrow\left(Y, \sigma_{1}, \sigma_{2}\right)$, the properties: $i j$-strongly $\theta$-continuous, $i j-S C, i j-\delta$-continuous, $i$-continuous and $i j$-almost continuous are equivalent.

PROOF. From Theorem 2.8 and Corollary 4.2.

THEOREM 4.2. For a mapping $f:\left(X, \tau_{1}, \tau_{2}\right) \longrightarrow\left(Y, \sigma_{1}, \sigma_{2}\right)$ the following implications hold: $j$-strongly continuous $\longrightarrow i j$-perfectly continuous and $i j$-completely continuous $\longrightarrow i j-R$ map $\longrightarrow$ $i j-\delta$-continuous.

PROOF. If $f$ is $j$-strongly continuous, then from Corlllary $4.1 f^{-1}(B)$ is both $j$-open and $j$-closed for every $B \subset Y$, hence $f$ is $i j$-perfectly continuous. Also, if $f$ is $i j$-completely continuous, let $V$ be $i j-r . o$ subset of $Y$, then $V$ is $i$-open and $f^{-1}(V)$ is $i j-r . o$. So $f$ is an $i j-R$ map.

Finally, if $f$ is an ij $-R$ map, then $f^{-1}(V)$ is $i j-r . o$ in $X$ for every $i j-r . o$ subset $V$ of $Y$, let $f^{-1}(V)=U$, since $f\left(f^{-1}(V)\right) \subset V$ so $f(U) \subset V$. Hence $f$ is $i j-\delta$-continuous.

REMARK 4.2. The converse of the above implications may not be true as seen from the following example:

Let $X=Y=\{a, b, c\}$ and let $\tau_{1}=\{\phi,\{a\},\{b\},\{a, b\}, X\}, \tau_{2}=\{\phi, X\}, \sigma_{1}=\{\phi, X\}, \sigma_{2}=$ $\{\phi,\{a\},\{c\},\{a, c\}, X\}$. Let $f:\left(X, \tau_{1}, \tau_{2}\right) \longrightarrow\left(Y, \sigma_{1}, \sigma_{2}\right)$ be the identity map. Then $f$ is 12-perfectly continuous but it is not 2-strongly continuous.

THEOREM 4.3. If $f:\left(X, \tau_{1}, \tau_{2}\right) \longrightarrow\left(Y, \sigma_{1}, \sigma_{2}\right)$ is $i j$-completely continuous, then it is $i j-S C$.

PROOF. Let $V$ be an $i$-open subset of of $Y$ and let $U=f^{-1}(V)$. Since $f$ is $i j$-completely continuous, so $f^{-1}(V)$ is $i j-r$.o and $f\left(f^{-1}(V)\right) \subset V$. Hence $f(U)=f(i-\operatorname{int}(j-c l U)) \subset V$. So $f$ is $i j-S C$.

REMARK 4.3. The converse of the above theorem may not be true as shown in the following example.

EXAMPLE 4.3. Consider Exmaple 4.1, $f$ is $i j-S C$ but it is not $i j$-completely continuous.

The following diagram gives us the relations between these kinds of continuity.

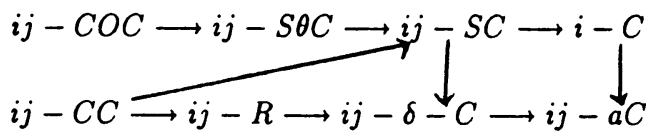

also: $j-S t C \longrightarrow i j-P C$.

where

$i j-C O C=i j$-clopen continuous, $i j-S \theta C=i j$-strongly $\theta$-continuous

$i-C=i$-continuous, $i j-C C=i j$-completely continuous, $i j-R=i j-R$ map

$i j-\delta C=i j-\delta$-continuous, $i j-a C=i j$-almost continuous,

$j-S t C=j$-strongly continuous, $i j-P C=i j$-perfectly continuous.

\section{REFERENCES}

1. Kelly, J.C. Bitopological Spaces, Proc. London Math. Soc. (3)13(1963), 71-89.

2. Munshi, B.M. and Bassan, D.S. Super-continuous mapping, Indian J. Pure Appl. Math. 13(2) (1982), 229-236.

3. Noiri, T. Super-continuity and some strong forms of continuity, Indian J. Pure Appl. Math. 15(3) $(1984), 241-250$.

4. Noiri, T. On $\delta$-continuous functions. $\underline{J}$. Korean Math. $\underline{\text { Soc. }} \underline{\text { 16 }}$ (1980), 161-166.

5. Arya, S.P. and Gupta, R. On strongly continuous mappings. Kyungpook Math. J. $14(1974)$, 131-143

6. Bose, S. and Sinha, D. Pairwise almost continuous map and weakly continuous map in bitopological spaces, Bull. Cal. Math. Soc. 4ㅡ(1982), 195-206. 
7. Banerjee, G.K. On pairwise almost strongly 0-continuous mappings, Bull. Cal. Math. Sioc 79(1987), 314-320.

8. Singal, A.R. and Arya, S.P. On pairwise almost regular spaces, Glasnzk Math. 6(26)(1971), 335-343.

9. Singal, M.K. and Singal, A.R. Some more separation axioms in bitiopological spaces. Ann. Son. Sci. Bruxelles. 84(1970), 207-230. 


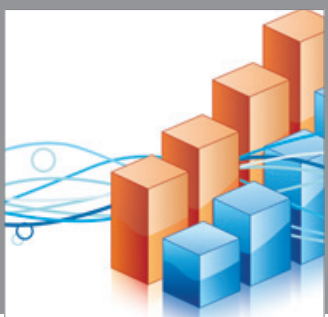

Advances in

Operations Research

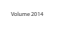

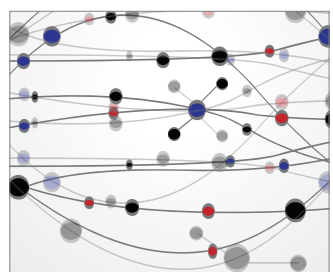

\section{The Scientific} World Journal
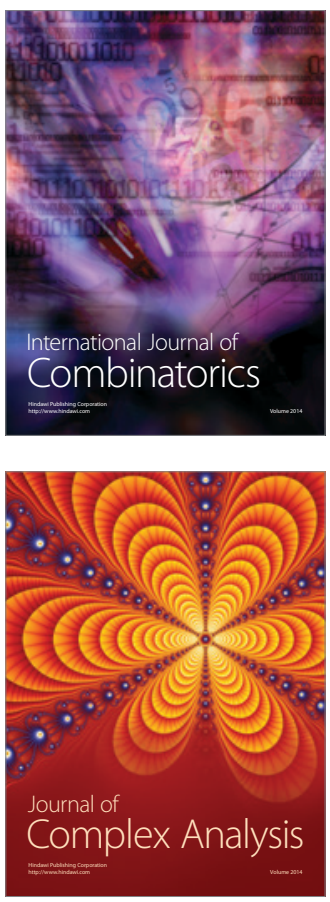

International Journal of

Mathematics and

Mathematical

Sciences
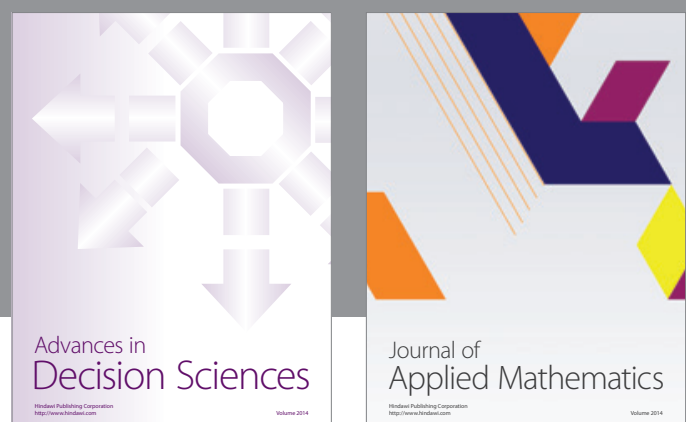

Journal of

Applied Mathematics
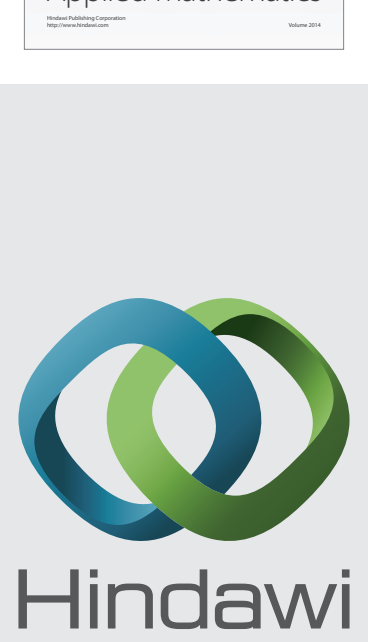

Submit your manuscripts at http://www.hindawi.com
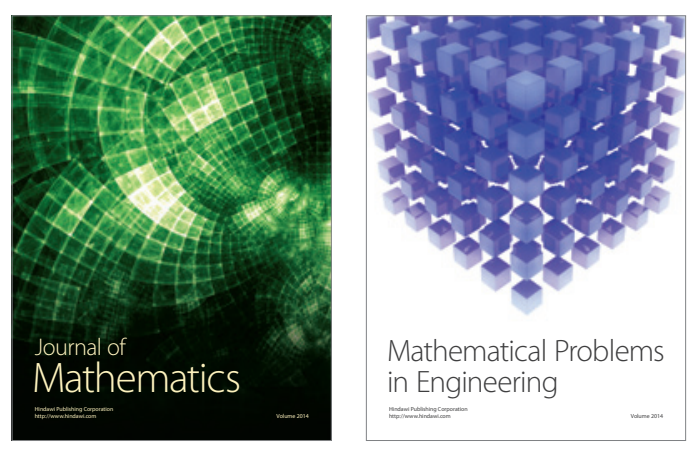

Mathematical Problems in Engineering
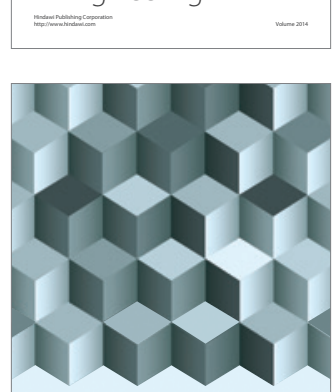

Journal of

Function Spaces
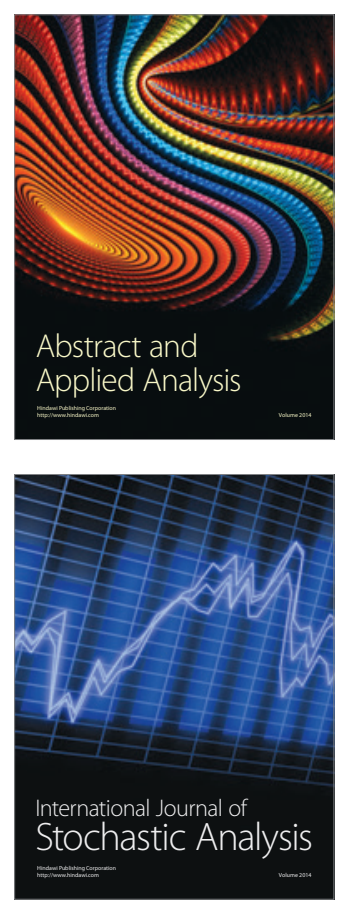

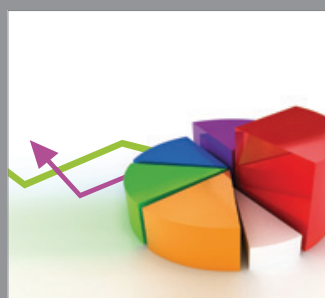

ournal of

Probability and Statistics

Promensencen
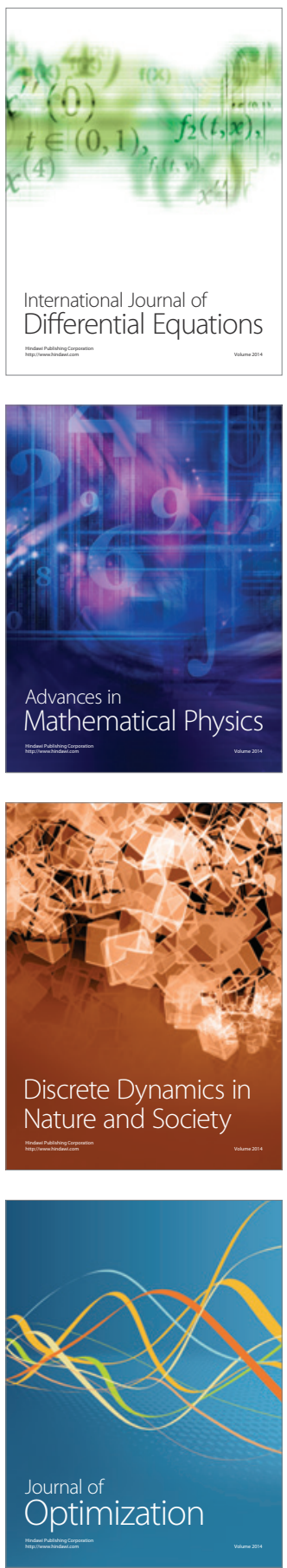